\section{Taxol: Women with ovarian cancer participate in a new clinical trial}

\author{
By Janie Brown, Barbara McDermott, Nancy Runzer
}

\begin{abstract}
Taxol has been hailed by some as a promising new chemotherapy drug for the treatment of women with ovarian cancer. An international study has been mounted to determine the optimal dose and the optimal infusion duration of Taxol.

Nurses on the in-patient chemotherapy unit at the B.C. Cancer Agency, Vancouver Clinic, have been actively involved in caring for women as they receive Taxol. This article offers clinical tips from the Vancouver experience about organizing treatment rooms, setting up $I V s$, administering the drug, monitoring the patient for side-effects and dealing with the many difficult questions asked by women involved in this clinical trial.
\end{abstract}

The British Columbia Cancer Agency (BCCA) is one of 22 Canadian centres participating in an international study of Taxol, a new drug for the treatment of women with ovarian cancer. As of March 1, 1992 a total of 43 patients have been randomized to the study at BCCA and a total of 145 treatments have been administered. Nurses on the in-patient chemotherapy unit have been actively involved in the care of women receiving Taxol. The purpose of this article is to share clinical experiences in relation to Taxol with other oncology nurses across Canada.

Cancer of the ovary was the fifth leading cause of cancer death in Canadian women in 1990 (Canadian Cancer Statistics, 1990). Chemotherapy regimens which contain either cisplatin or carboplatin improve survival in these patients, although about half of them will eventually relapse (Omura, Blessing \& Ehrlich, 1986). Re-treatment with one of the two platinum-based drugs can produce further response, but there are still no useful forms of

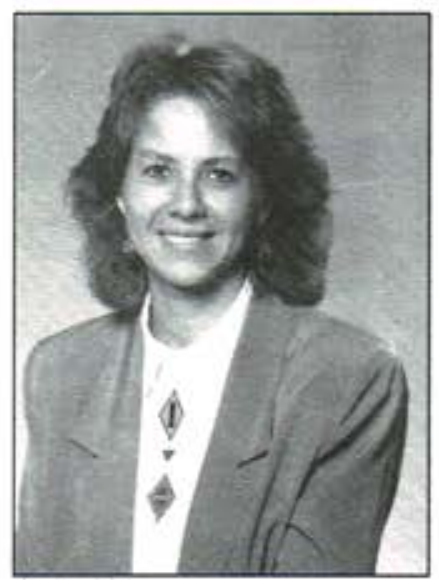

Janie Brown

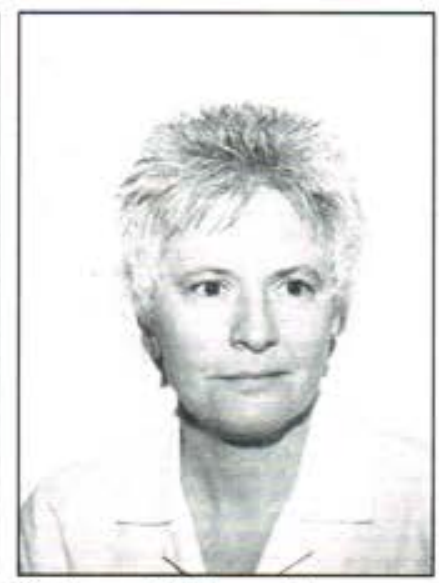

Barbara McDermott

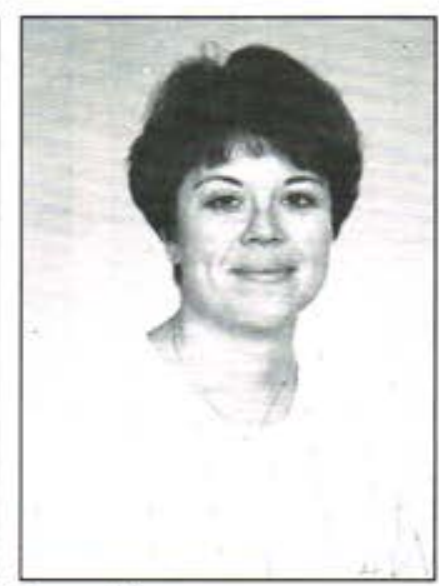

Nancy Runzer therapy for those patients whose disease progresses despite treatment. Taxol may prove to be a helpful drug for some of these patients.

Taxol is a new cytotoxic agent isolated from the bark of the Pacific Yew tree (Taxus brevifolia). Phase I and II trials have indicated that Taxol has major potential in the treatment of ovarian cancer (McGuire et al., 1989). Response rates of $21 \%$ to $37 \%$ have been demonstrated in previously treated patients, many of whom were refractory to cisplatin (McGuire et al., 1989). Several responses have been complete and long-term. Dose-limiting toxicities are neutropenia, peripheral neuropathy and hypersensitivity reactions.

\section{The Study}

The purpose of this international study is to determine the optimal dose and optimal infusion duration of Taxol (GO OV TAX, 1991). Eligible patients are therefore randomized to one of four study arms: Taxol $175 \mathrm{mg} / \mathrm{m} 2$ or Taxol $135 \mathrm{mg} / \mathrm{m} 2$ administered over either three hours or 24 hours. After receiving two treatments three weeks apart patients are assessed for disease response. If the disease has progressed after two treatments the patient goes off the study; if the disease is stable or there is a partial or complete response the patient will receive at least four more treatments, unless there is relapse or unacceptable toxicity during that time. The decision may be made to continue after six treatments depending on disease status.

\section{Our experience at the Vancouver clinic}

The Taxol patient

Many of the women selected for the Taxol study have waited a long time. They were often aware for many months that Taxol would

\section{L'EXPÉRIENCE DU TAXOL: VANCOUVER}

\section{ABRÉGÉ}

Le Taxol a été accueilli par certains comme étant un nouveau produit de chimiothérapie très prometteur pour le traitement du cancer de l'ovaire. Une étude internationale a été mise sur pied pour déterminer la dose et la durée de perfusion optimales du Taxol.

A la clinique de Vancouver de l'Agence du Cancer de la C.-B., les infirmières du service de consultations internes de chimiothérapie ont participé activement aux soins de femmes recevant du Taxol. Elles parlent de leur expérience et donnent de nombreux "tuyaux" cliniques sur la préparation des infirmières, l'organisation des salles de traitement, la mise en place des perfusions, l'administration du médicament et la surveillance des patientes afin de pouvoir déceler les effets secondaires. Elles en décrivent aussi les effets secondaires typiques et explorent quelques-unes des inquiétudes que partagent un grand nombre de femmes participant à des essais cliniques.

Janie Brown, RN, MA, MSN (candidate) is clinical nurse specialist, Barbara McDermott, RN is nursing manager and Nancy Runzer, $\mathrm{RN}$ is clinical instructor on the chemotherapy unit of the British Columbia Cancer Agency, Vancouver Clinic. 
eventually become available for the treatment of ovarian cancer and knew that they might or they might not be eligible for the study. Boosted by recent media-hype and their acceptance into the study, many women come to the in-patient chemotherapy unit for their first treatment feeling both excited and fearful. They are excited by the fact that Taxol has been heralded by some as a new wonder-drug for cancer of the ovary, and they feel lucky to be selected as one of only 300 women internationally who will receive the drug. Along with that excitement, however, they may also feel afraid and anxious about being involved in a clinical trial and receiving a drug which bas not yet been approved for general use in the treatment of cancer. All of the women are aware they will receive just two treatments before they are assessed for tumour response, and that if they have progressive disease at that point they will be taken off the protocol. This creates a lot of anxiety for many women as they anticipate the results of their disease status reassessment. During admission for their second treatment this anxiety is most evident. Coming off protocol may shatter their dream of a cure and instill in some women a sense of hopelessness about the future.

As nurses, we field many questions from these women about their involvement in the Taxol study. "Perhaps I would have been better off to be on the higher dose"; "Perhaps the dose isn't high enough to do its job on the cancer"; "Can I receive more than six treatments if it is working for me?"; "What if supplies run out, can I go to the States to receive the drug?"; "What if it doesn't work and 1 have put all my hopes into this miracle drug?" These are difficult questions to answer, and demand that nurses take all the time that is required to help women work through some of their concerns related to being involved in this clinical trial.

\section{The treatment}

\section{Room preparation}

The resuscitation/emergency cart is placed outside the patient's room for the first hour of infusion. An emergency drug tray is set up

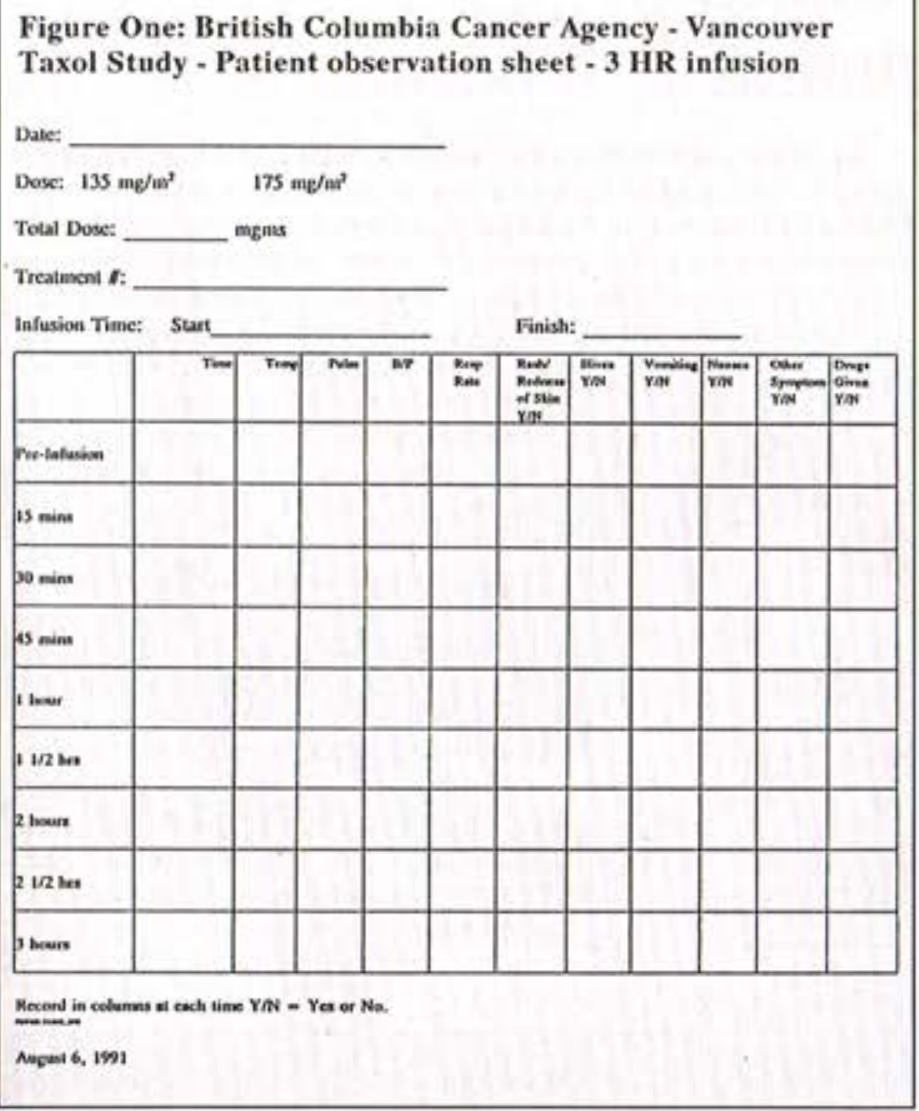

in the room in the event of an acute hypersensitivity reaction. The tray consists of specific drugs clearly outlined in the protocol. A copy of the instructions for management of acute hypersensitivity reactions, from the protocol, is pinned on the wall for quick reference in an emergency situation. Oxygen and suction equipment are at the bedside of each patient. A three-hour or 24-hour observation sheet (Figure One) and a fluid balance sheet are also placed in the room for each patient.

\section{IV set-up}

IV equipment is set up as in Figure Two. Vented nitroglycerine tubing (A) for volumetric pumps, with Luer-lock adapter is primed with normal saline and attached to a nitroglycerine extension set with a 0.22 micron, 72 -hour filter. A solution set with two injection sites (B) is primed with normal saline and attached at the lowest port of $\mathrm{A}$.

Nitroglycerine tubing is required because the cremaphor, the substance with which Taxol is dissolved, leaches plasticizer from the polyvinylchloride (PVC) of regular IV tubing.

The PVC extension tubing attached to a Port-a-Cath Gripper needlc cannot be used for the same reason. The nitroglycerine extension tubing should be connected directly to a regular Huber-point needle.

\section{Administration of Taxol}

Patients are premedicated according to the protocol with dexamethasone $20 \mathrm{mg}$ po (at home), 12 hours and six hours prior to the infusion of Taxol, and diphenhydramine $50 \mathrm{mg}$ IV and ranitidine $50 \mathrm{mg}$ IV, 30 minutes prior to the Taxol infusion. Premedication is essential in an attempt to avoid an acute hypersensitivity reaction. Taxol is administered as a continuous infusion in a $500 \mathrm{~mL}$ glass bottle of $5 \%$ dextrose or normal saline over three hours, or in two $500 \mathrm{~mL}$ bottles over 12 hours each, for the 24 -hour infusion. The first $18 \mathrm{~mL}$ of IV solution (volume of the IV set) are infused at a fast rate $(300 \mathrm{~mL} / \mathrm{h})$ in the previously primed line, to ensure that the Taxol has reached the end of the IV tubing. This procedure enables

Figure Two: Equipment set-up

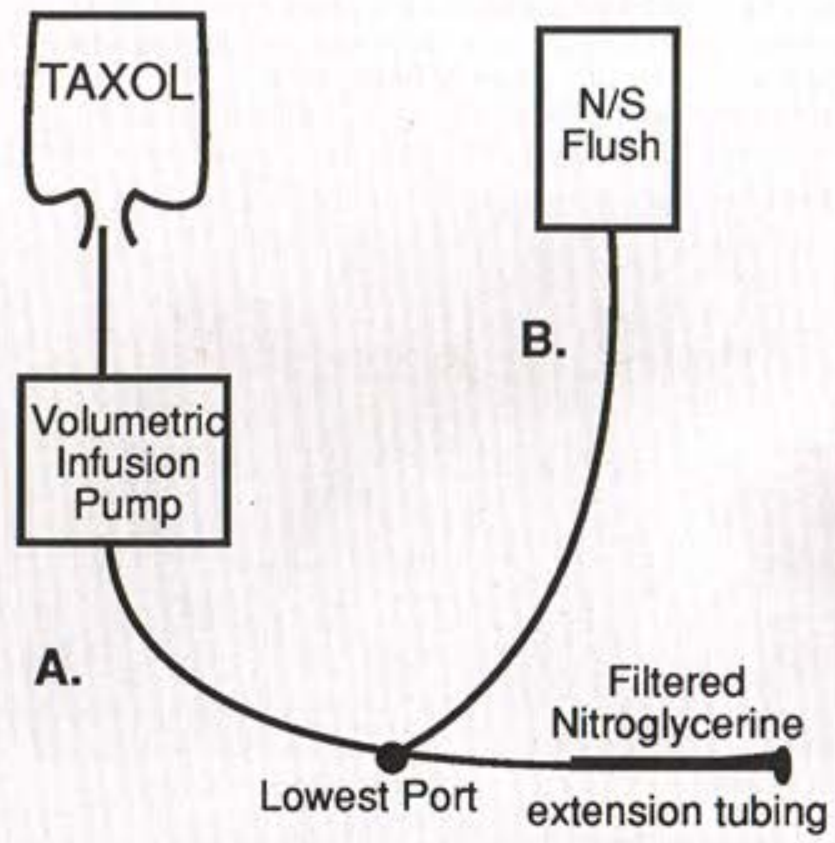


the nurse to accurately assess the patient as she begins to receive the Taxol and hence to closely monitor for the development of an acute hypersensitivity reaction.

\section{Monitoring the patient}

Patient observation sheets were designed for both the three-hour and the 24-hour infusions (Figure One).

The nurse must remain with the patient for the first hour of the infusion. Temperature, pulse, blood pressure and respirations are taken pre-infusion, every 15 minutes for the first hour, every 30 minutes for the next two hours, hourly for the next four hours and two hourly until the end of the infusion if receiving the 24-hour infusion. Other observations are also documented, rash/redness of skin, hives, vomiting, nausea, and any other symptoms. This documentation assists in identifying patterns in side-effects experienced by the patients while in hospital. Patients do not

Table One: Toxicities documented from other Taxol studies

\section{Acute hypersensitivity reactions}

Hypersensitivity reactions are thought to be caused by the solvent Cremaphor EL in which Taxol is dissolved (Weiss, Donehover, \& Wiernik, 1990). Weiss et al (1990) found that out of 32 patients who had reacted, $78 \%$ did so within the first 10 minutes of the infusion. One reaction was fatal and the rest were reversible with treatment. Intensive premedication with antihistamines, steroids and $\mathrm{H}-2$ receptor antagonists decreases the incidence of reactions (Weiss et al., 1990). It is not yet clear whether increasing the duration of infusion of the drug also decreases the likelihood of reaction.

\section{Neutropenia}

Neutropenia was the major dose limiting factor in phase I trials. The nadir is around day eight and the duration of neutropenia is generally short (days 5-15) (McGuire et al., 1990). Neutropenia is non-cumulative over successive treatments and rarely demands a delay in scheduled treatment.

\section{Neurotoxicity}

Neurotoxicity rarely occurs with dosages less than $170 \mathrm{mg} / \mathrm{m} 2$. When it does, symptoms include numbness and parasthesias of the soles of the feet and fingers, both of which resolve within several months after discontinuation of treatment (Rowinsky et al., 1991).

\section{Cardiac arrythmias}

Asymptomatic bradycardia has been noted during the infusion of Taxol in $29 \%$ of patients in one study with two more serious bradyarrythmias in two patients (McGuire, 1990).

\section{Alopecia}

All patients develop a sudden and total loss of scalp hair after one or two courses and a cumulative loss of facial and body hair with successive treatments (Rowinsky et al., 1991).

\section{Joint discomfort and myalgias}

Joint and muscle discomfort may occur two to three days after the Taxol infusion and generally subside within four to seven days (Rowinsky et al., 1991). This discomfort was described by some to be the most distressing side-effect from Taxol (McGuire et al., 1989).

\section{Nausea and vomiting}

Gastro-intestinal toxicity is mild to moderate (McGuire et al., 1989).

\section{Local venous irritation}

Extravasation of Taxol causes swelling, erythema and mild pain in the area of infiltration which subsides within five to ten days without ulceration of the skin (McGuire et al., 1989). monitor vital signs or side-effects on a standard report form while at home and therefore there is no systematic record of the severity of side-effects experienced by patients after discharge.

\section{Side-effects experienced by patients at the Vancouver clinic}

\section{Acute hypersensitivity reactions}

To date, only two patients have experienced acute hypersensitivity reactions. Both reactions occurred on the second treatment and within the first one $\mathrm{mL}$ of infused Taxol. Both patients complained of tightness in the throat and across the chest with some difficulty breathing. The Taxol was immediately discontinued and normal saline infused to maintain IV access. The procedure (from the protocol) for the management of a hypersensitivity reaction was followed for one patient. The patient received epinephrine, diphenhydramine and methylprednisolone IV with a recovery from the symptoms within a few minutes. It was decided by the principle investigator of the study that the infusion should be restarted with close observation. The patient subsequently received the complete dose with no further problems and went on to receive four more cycles of Taxol uneventfully. The second patient did not require any medications as the reaction subsided within a minute of discontinuing the Taxol. The Taxol was restarted soon after with no further difficulty.

\section{Nausea and vomiting/diarrhea}

Only one patient on the three-hour infusion experienced nausea while in hospital. Twenty-four per cent (11/45) of the patients on the 24-hour infusion, however, complained of nausea and/or vomiting. This most often began after the fifth hour of infusion up until 24 hours after the infusion was commenced. The dose of Taxol was not related to the incidence or severity of nausea. Most patients were treated with dimenhydrinate $50 \mathrm{mg} I V$ with good effect. Eight of the 45 patients receiving the 24 -hour infusion experienced abdominal cramping and/or diarrhea, seven or more hours into the infusion.

\section{Asymptomatic bradycardia}

About half the patients in the 24-hour group demonstrated bradycardia, with a drop in baseline heart rate by $10-20$ beats/minute. All the bradycardias were asymptomatic and no heart rate dropped below 50 beats/minute. No patients in the three-hour group were bradycardic.

\section{Facial flushing}

One-third of patients experienced marked facial flushing beginning anywhere from one hour into the infusion onwards. This subsided within the 24 hours following the infusion.

\section{Total body alopecia}

All patients except one have experienced total scalp hair loss after the first or second treatments, and most have lost eyebrows and other body hair with subsequent treatments.

\section{Neutropenia}

Only one patient has had a dose reduction because of extremely low neutrophil counts. No patient has required a delay in treatment.

\section{Joint and muscle pain}

Several patients have complained of muscle weakness a few days after discharge, particularly in the lower legs, but this has rarely been described as painful or distressing. One patient reports taking oral calcium tablets which she believes are helpful in relieving muscle cramps/weakness in her lower legs.

\section{Neurotoxicity}

Several patients have described numbness on the soles of the feet and occasionally on the tips of the fingers. This has not commonly been described as problematic. 


\section{Local venous irritation}

Two patients have experienced local irritation of the skin due to extravasation of Taxol. No research has been documented as to the treatment for this type of extravasation. Based on experience with vesicant extravasations we recommend topical application of ice to the area for 15-20 minutes each hour for four hours, and close observation of the site by the patient after discharge. The two patients experienced slight swelling, redness and mild pain which took five to seven days to resolve.

\section{Conclusion}

After 300 women have been accrued internationally, findings will be analyzed and conclusions drawn about the most effective dosages and infusion times for Taxol. It is not possible at this time therefore to judge whether Taxol will prove to be the promised wonder-drug of the 1990s.
It has been, and continues to be, a challenge for nurses caring for women receiving Taxol. Women must contend with some difficult issues around being involved in a clinical trial, they must deal with some distressing side-effects of treatment and they must ultimately live with the uncertainty that Taxol may or may not offer them some hope for the future.

\section{Acknowledgements}

The authors would like to thank the nurses of 6 East for their valuable contribution to this article and for the excellent nursing care they provide to all patients requiring in-patient chemotherapy.

The authors would be pleased to discuss the care of women receiving Taxol and share printed resources ie, care plans for patient education, acute hypersensitivity reactions and extravasation procedures.

They may be contacted through the chemotherapy unit at (604) 877-6000 extension 2371.

\section{References}

1. GO OV TAX. A multicenter randomized comparative study of Taxol in platinum treated ovarian cancer (high vs low dose; long vs short duration). Principle investigator: Swenerton, Kenneth: B.C. Cancer Agency, 1991.

2. McGuire, W.P., Rowinsky, E.K., Rosenhein, N.B., Grumbine, F.C., Ettinger, D.S., Armstrong, D.K., \& Donehower, R.C. Taxol: A unique antineoplastic agent with significant activity in advanced ovarian epithelial neoplasms. Annals of Internal Medicine, 111, $1989,273-279$.

3. National Institute of Canada. Canadian Cancer Statistics. Toronto: Author, 1990.

4. Omura, G., Blessing, J.A., \& Ehrlich, C.E. A randomized trial of
Cyclophosphamide and Doxorubicin with or without Cisplatin in advanced ovarian carcinoma: A gynecologic oncology group study. Cancer, 57, 1986, 1725-1730.

5. Rowinsky, E.K., Gilbert, M.R., McGuire, W.P., Noe, D.A. Grochow, L.B., Forastiere, A.A., Ettinger, D.S., Lubejko, B.G., Clark, B., Sartorius, S.E., Cornblath, D.R., Hendricks, C.B., Donehower, R.C. Sequences of Taxol and Cisplatin: A phase I and pharmacologic study. Journal of Clinical Oncology, 9, (9), 1692-1703, 1991.

6. Weiss, R.B., Donehover, R.C., \& Wernik, P.H. Hypersensitivity reactions from Taxol. Journal of Clinical Oncology, 8, 1263-1268, 1990.

\section{Cancer's a Funny Thing}

I wish I had the voice of Homer

To sing of rectal carcinoma,

Which kills a lot more chaps, in fact,

Than were bumped off when Troy was sacked.

I noticed I was passing blood (Only a few drops, not a flood)

So pausing on my homeward way

From Tallahassee to Bombay

I asked a doctor, now my friend,

To peer into my hinder end,

To prove or to disprove the rumour

That I had a malignant tumour.

They pumped in $\mathrm{Ba} \mathrm{SO}_{4}$

Till I could really stand no more,

And, when sufficient had been pressed in,

They photographed my large intestine.

In order to decide the issue

They next scraped out some bits of tissue.

(Before they did so, some good pal

Had knocked me out with pentothal,

Whose action is extremely quick,

And does not leave me feeling sick.)

The microscope returned the answer

That I had certainly got cancer.

So I was wheeled to the theatre

Where holes were made to make me better.
One set is in my perineum

Where I can feel, but can't yet see 'em.

Another made me like a kipper

Or female prey of Jack the Ripper.

Through this incision, I don't doubt,

The neoplasm was taken out, Along with colon, and lymph nodes

Where cancer cells might find abodes.

A third much smaller hole is meant

To function as a ventral vent:

So now I am like two-faced Janus

The only ${ }^{1}$ god who sees his anus.

I'll swear, without the risk of perjury,

It was a snappy bit of surgery.

My rectum is a serious loss to me,

But I've a very neat colostomy,

And hope, as soon as I am able,

To make it keep a fixed time-table.

So do not wait for aches and pains To have a surgeon mend your drains; If he says "cancer" you're a dunce Unless you have it out at once, For if you wait it's sure to swell, And may have progeny as well.

1. In India there are several more With extra faces, up to four, But both in Brahma and in Shiva, I own myself an unbeliever.
My final word, before I'm done, Is "Cancer can be rather fun."

Thanks to the nurses and Nye Bevan

The NHS is quite like heaven

Provided one confronts the tumour

With a sufficient sense of humour.

I know that cancer often kills,

But so do cars and sleeping pills;

And it can hurt one till one sweats,

So can bad teeth and unpaid debts.

A spot of laughter, I am sure,

Often accelerates one's cure;

So let us patients do our bit

To help the surgeons make us fit.

J.B.S. Haldane

1892 - 1964

"The main functions of my rhyme", wrote Professor Haldane, who died in 1964 at the age of 73, were "to induce cancer patients to be operated on early and to be cheerful about it."

From Writing in England Today, Karl Miller (ed.), Penguin, 1968. 